\title{
Partitions with designated summands
}

\author{
by \\ G. E. Andrews (University Park, PA), R. P. Lewis (Brighton) \\ and J. LoveJoy (Paris)
}

1. Introduction. The object of our study is partitions with designated summands. They are constructed by taking ordinary partitions and tagging exactly one of each part size. Thus there are 15 partitions of 5 with designated summands:

$$
5^{\prime}, 4^{\prime}+1^{\prime}, 3^{\prime}+2^{\prime}, 3^{\prime}+1^{\prime}+1,3^{\prime}+1+1^{\prime}, 2^{\prime}+2+1^{\prime},
$$

$2+2^{\prime}+1^{\prime}, 2^{\prime}+1^{\prime}+1+1,2^{\prime}+1+1^{\prime}+1,2^{\prime}+1+1+1^{\prime}, 1^{\prime}+1+1+1+1$,

$1+1^{\prime}+1+1+1,1+1+1^{\prime}+1+1,1+1+1+1^{\prime}+1,1+1+1+1+1^{\prime}$.

The total number of partitions of $n$ with designated summands is denoted by $\operatorname{PD}(n)$. Hence $\operatorname{PD}(5)=15$.

We shall also study $\operatorname{PDO}(n)$, the total number of partitions of $n$ with designated summands in which all parts are odd. Thus $\operatorname{PDO}(5)=8$.

While these objects have not appeared in the literature before, they are tacitly considered by P. A. MacMahon [13] in his work on generalized divisor sums. Indeed, MacMahon's $A_{n, k}$ is the number of partitions of $n$ with designated summands wherein exactly $k$ different magnitudes occur among all the parts. MacMahon [13, Section 17] is able to connect $A_{n, k}$ with numerous divisor sum identities due to Glaisher [7], Ramanujan [15] and others. However, MacMahon neglected the very interesting case wherein the number of different magnitudes of parts is suppressed. We shall consider the generating functions:

$$
\operatorname{pd}(q)=\sum_{n=0}^{\infty} \operatorname{PD}(n) q^{n},
$$

and

$$
\operatorname{pdo}(q)=\sum_{n=0}^{\infty} \operatorname{PDO}(n) q^{n} .
$$

2000 Mathematics Subject Classification: 05A17, 11P81. 
In Section 2, we shall show (among other things) that

$$
\operatorname{pd}(q)=\frac{\eta(6 z)}{\eta(z) \eta(2 z) \eta(3 z)},
$$

where

$$
\eta(z)=q^{1 / 24} \prod_{n=1}^{\infty}\left(1-q^{n}\right),
$$

and

$$
q:=e^{2 \pi i z}
$$

Also

$$
\operatorname{pdo}(q)=\frac{\eta(4 z) \eta^{2}(6 z)}{\eta(z) \eta(3 z) \eta(12 z)} .
$$

We examine $q$-series identities in Section 3 , and in Section 4 we relate $\operatorname{PD}(n)$ and $\operatorname{PDO}(n)$ to several well-known partition functions. As a result, we can easily deduce that $\operatorname{PDO}(n)$ is even unless either $n$ is 0 or $n$ is a perfect square not divisible by 3 .

Section 5 is devoted to arithmetic properties of partitions with designated summands and in Section 6 we investigate some modular relations.

\section{The generating functions}

Theorem 1. Suppose $S$ is a set of positive integers. Denote by $\operatorname{PD}_{S}(n)$ the number of partitions of $n$ with designated summands all of whose parts lie in $S$, and denote by $\operatorname{pd}_{S}(q)$ the generating function for $\operatorname{PD}_{S}(n)$. Then

$$
\operatorname{pd}_{S}(q)=\prod_{n \in S} \frac{1-q^{6 n}}{\left(1-q^{n}\right)\left(1-q^{2 n}\right)\left(1-q^{3 n}\right)} .
$$

Proof. We see immediately that

$$
\begin{aligned}
\operatorname{pd}_{S}(q) & =\prod_{n \in S}\left(1+q^{n}+2 q^{2 n}+3 q^{3 n}+4 q^{4 n}+\ldots\right) \\
& =\prod_{n \in S}\left(1+\frac{q^{n}}{\left(1-q^{n}\right)^{2}}\right)=\prod_{n \in S} \frac{1-q^{n}+q^{2 n}}{\left(1-q^{n}\right)^{2}} \\
& =\prod_{n \in S} \frac{1+q^{3 n}}{\left(1-q^{n}\right)^{2}\left(1+q^{n}\right)}=\prod_{n \in S} \frac{1-q^{6 n}}{\left(1-q^{n}\right)\left(1-q^{2 n}\right)\left(1-q^{3 n}\right)}
\end{aligned}
$$

Corollary 2. Equality (1.3) is valid.

Proof. This is the case $S=\mathbb{N}$, the set of all positive integers. 
COROllary 3. If $S_{k}$ is the set of all positive integers not divisible by $k$, then

$$
\operatorname{pd}_{S_{k}}(q)=\operatorname{pd}(q) / \operatorname{pd}\left(q^{k}\right) .
$$

Proof. This assertion is the specialization of Theorem 1 to $S_{k}$.

Corollary 4. Equality (1.6) is valid.

Proof. This follows directly from Corollary 3 with $k=2$.

Indeed we might note that if $S$ consists of all positive integers congruent to one of $a_{1}, \ldots, a_{j}$ modulo $k$, and if $S$ is symmetric (i.e. whenever $\alpha \in S$ then all positive integers $\equiv-\alpha(\bmod k)$ are in $S)$, then $\operatorname{pd}_{S}(q)$ is a modular function. The subsequent sections are restricted to the instances of $\operatorname{pd}_{S}(q)$ that seem to have the most interest, namely $\operatorname{pd}(q)$ and $\operatorname{pdo}(q)$.

3. $q$-Series for $\operatorname{pd}(q)$ and $\operatorname{pdo}(q)$. There are several reasons for considering $q$-series expansions of these generating functions. Often such expansions provide efficient algorithms for the calculation of their coefficients. This turns out to be the case for both $\operatorname{pd}(q)$ (see (3.4)) and $\operatorname{pdo}(q)$ (see (3.13)). In addition, we occasionally uncover new mysteries. In our present study, it is quite mysterious that the $q$-series (3.3) and (3.12) which seem to have complex coefficients, in fact do not.

Theorem 5. For $|q|<1$ and $\zeta=e^{\pi i / 3}$,

$$
\begin{aligned}
\operatorname{pd}(q) & =\frac{(\zeta q ; q)_{\infty}\left(\zeta^{-1} q ; q\right)_{\infty}}{(q ; q)_{\infty}^{2}} \\
& =1+\sum_{n=1}^{\infty} \frac{\left(-q^{3} ; q^{3}\right)_{n-1} q^{n}}{(q ; q)_{n}\left(q^{2} ; q^{2}\right)_{n-1}\left(1-q^{n}\right)} \\
& =1+\zeta \sum_{n=1}^{\infty}\left(\frac{(\zeta q ; q)_{n-1}}{(q ; q)_{n}}\right)^{3}\left(1-\zeta q^{2 n}\right)(-1)^{n} q^{\left(\begin{array}{c}
n+1 \\
2
\end{array}\right)} \zeta^{-n} \\
& =\frac{\sum_{n=0}^{\infty} q^{\left(\begin{array}{c}
n+1 \\
2
\end{array}\right)}-3 \sum_{n=0}^{\infty} q^{\left(\begin{array}{c}
3 n+2 \\
2
\end{array}\right)}}{\sum_{n=0}^{\infty}(-1)^{n}(2 n+1) q^{\left(\begin{array}{c}
n+1 \\
2
\end{array}\right)}}
\end{aligned}
$$

where

$$
(a ; q)_{n}=(1-a)(1-a q) \ldots\left(1-a q^{n-1}\right) .
$$

Proof. By (1.3),

$$
\begin{aligned}
\operatorname{pd}(q) & =\frac{\left(q^{6} ; q^{6}\right)_{\infty}}{(q ; q)_{\infty}\left(q^{2} ; q^{2}\right)_{\infty}\left(q^{3} ; q^{3}\right)_{\infty}}=\frac{\left(-q^{3} ; q^{3}\right)_{\infty}}{(q ; q)_{\infty}^{2}(-q ; q)_{\infty}} \\
& =\frac{(\zeta q ; q)_{\infty}\left(\zeta^{-1} q ; q\right)_{\infty}}{(q ; q)_{\infty}^{2}},
\end{aligned}
$$


which proves (3.1). Identity (3.2) may be rewritten as follows:

$$
\sum_{n=0}^{\infty} \frac{(\zeta ; q)_{n}\left(\zeta^{-1} ; q\right)_{n}}{(q ; q)_{n}(q ; q)_{n}}=\frac{(\zeta q ; q)_{\infty}\left(\zeta^{-1} q ; q\right)_{\infty}}{(q ; q)_{\infty}(q ; q)_{\infty}}
$$

and in this form we see that it is a specialization of the $q$-analog of Gauss's theorem [2, p. 20, Cor. 2.4, $\left.a=\zeta, b=\zeta^{-1}, c=q\right]$. Identity (3.3) may be rewritten as follows:

(3.7) $\lim _{t \rightarrow 0} \sum_{n=0}^{\infty} \frac{(\zeta ; q)_{n}^{3}}{(q ; q)_{n}^{3}} \cdot \frac{1-\zeta q^{2 n}}{1-\zeta} \cdot \frac{\left(t^{-1} ; q\right)_{n}}{(\zeta q t ; q)_{n}}\left(\frac{t \zeta q}{\zeta^{2}}\right)^{n}=\frac{\left(\zeta^{-1} q ; q\right)_{\infty}(\zeta q ; q)_{\infty}}{(q ; q)_{\infty}(q ; q)_{\infty}}$

and in this form we see that it is a specialization of the limiting form of Jackson's theorem [6, p. 238, (II.20)].

To obtain (3.4), we first note that

$$
\frac{(-1)^{n}\left(\zeta^{-n}-\zeta^{n+1}\right)}{1-\zeta}= \begin{cases}1 & \text { if } n \equiv 0(\bmod 3), \\ -2 & \text { if } n \equiv 1(\bmod 3), \\ 1 & \text { if } n \equiv 2(\bmod 3) .\end{cases}
$$

Hence by Jacobi's triple product [6, p. 239, (II.28)],

$$
\begin{aligned}
\frac{(\zeta q ; q)_{\infty}\left(\zeta^{-1} q ; q\right)_{\infty}}{(q ; q)_{\infty}^{2}} & =\frac{(q ; q)_{\infty}(\zeta ; q)_{\infty}\left(\zeta^{-1} q ; q\right)_{\infty}}{(1-\zeta)(q ; q)_{\infty}^{3}} \\
& =\frac{\sum_{n=0}^{\infty}(-1)^{n} q^{\left(\begin{array}{c}
n+1 \\
2
\end{array}\right)} \zeta^{-n} /(1-\zeta)}{(q ; q)_{\infty}^{3}} \\
& =\frac{\sum_{n=0}^{\infty} q^{\left(\begin{array}{c}
n+1 \\
2
\end{array}\right)}\left((-1)^{n}\left(\zeta^{-n}-\zeta^{n+1}\right) /(1-\zeta)\right)}{\sum_{n=0}^{\infty}(-1)^{n}(2 n+1) q^{\left(\begin{array}{c}
n+1 \\
2
\end{array}\right)}} \\
& =\frac{(\text { by }[9, \text { p. } 285, \text { Th. 357] })}{\sum_{n=0}^{\infty} q^{\left(\begin{array}{c}
n+1 \\
2
\end{array}\right)}-3 \sum_{n=0}^{\infty} q^{\left(\begin{array}{c}
(3+2 \\
2
\end{array}\right)}} .
\end{aligned}
$$

From (3.4) we immediately deduce a computationally fast recurrence for $\mathrm{PD}(n)$.

Corollary 6. For $n \geq 0$,

$$
\begin{aligned}
\sum_{j \geq 0} \operatorname{PD}\left(n-\left(\begin{array}{c}
j+1 \\
2
\end{array}\right)\right)(-1)^{j}(2 j+1) \\
\quad= \begin{cases}0 & \text { if } n \neq \text { triangular number }, \\
1 & \text { if } n=\left(\begin{array}{c}
m+1 \\
2
\end{array}\right) \text { and } m \neq 1(\bmod 3), \\
-2 & \text { otherwise. }\end{cases}
\end{aligned}
$$

Corollary 7. $\operatorname{PD}(3 n+2) \equiv 0(\bmod 3)$. 
Proof. By (3.4), we see that

$$
\operatorname{pd}(q) \equiv \frac{\sum_{n=0}^{\infty} q^{\left(\begin{array}{c}
n+1 \\
2
\end{array}\right)}}{(q ; q)_{\infty}^{3}}(\bmod 3) \equiv \frac{\sum_{n=0}^{\infty} q^{\left(\begin{array}{c}
n+1 \\
2
\end{array}\right)}}{\left(q^{3} ; q^{3}\right)_{\infty}}(\bmod 3)
$$

but this last expression has no powers of $q \equiv 2(\bmod 3)$ in its expansion.

Theorem 8. For $|q|<1$ and $\zeta=e^{\pi i / 3}$,

$$
\begin{aligned}
\operatorname{pdo}(q) & =\frac{\left(\zeta q ; q^{2}\right)_{\infty}\left(\zeta^{-1} q ; q^{2}\right)_{\infty}}{\left(q ; q^{2}\right)_{\infty}^{2}} \\
& =1+\sum_{n=1}^{\infty} \frac{\left(-q^{6} ; q^{6}\right)_{n-1} q^{n}}{(q ; q)_{2 n}\left(-q^{2} ; q^{2}\right)_{n-1}} \\
& =1-\zeta \sum_{n=1}^{\infty} \frac{(\zeta q ; q)_{2 n-1}\left(\zeta q^{2} ; q^{2}\right)_{n-1}(-1)^{n} q^{n^{2}}}{(q ; q)_{2 n}\left(q ; q^{2}\right)_{n}} \\
& =\frac{\sum_{n=-\infty}^{\infty} q^{(3 n)^{2}}-\sum_{n=-\infty}^{\infty} q^{(3 n+1)^{2}}}{1+2 \sum_{n=1}^{\infty}(-1)^{n} q^{n^{2}}} .
\end{aligned}
$$

Proof. By Corollary 3 with $k=2$

$$
\begin{aligned}
\operatorname{pdo}(q) & =\operatorname{pd}(q) / \operatorname{pd}\left(q^{2}\right) \\
& =\frac{(\zeta q ; q)_{\infty}\left(\zeta^{-1} q ; q\right)_{\infty}}{(q ; q)_{\infty}^{2}} \cdot \frac{\left(q^{2} ; q^{2}\right)_{\infty}}{\left(\zeta q^{2} ; q^{2}\right)_{\infty}\left(\zeta^{-1} q^{2} ; q^{2}\right)_{\infty}} \\
& =\frac{\left(\zeta q ; q^{2}\right)_{\infty}\left(\zeta^{-1} q ; q^{2}\right)_{\infty}}{\left(q ; q^{2}\right)_{\infty}^{2}} .
\end{aligned}
$$

Identity (3.11) may be rewritten as follows:

$$
\sum_{n=0}^{\infty} \frac{\left(\zeta ; q^{2}\right)_{n}\left(\zeta^{-1} ; q^{2}\right)_{n}}{\left(q^{2} ; q^{2}\right)_{n}\left(q ; q^{2}\right)_{n}} q^{n}=\frac{\left(\zeta^{-1} q ; q^{2}\right)_{\infty}\left(\zeta q ; q^{2}\right)_{\infty}}{\left(q ; q^{2}\right)_{\infty}\left(q ; q^{2}\right)_{\infty}},
$$

and in this form we see that it is a specialization of the $q$-analog of Gauss's theorem [2, p. 20, Cor. 2.4, $q$ replaced by $\left.q^{2}, a=\zeta, b=\zeta^{-1}, c=q\right]$.

Identity (3.12) may be rewritten as follows:

$$
\begin{aligned}
\lim _{t \rightarrow 0} \sum_{n=0}^{\infty} \frac{\left(\zeta / q ; q^{2}\right)_{n}}{\left(q^{2} ; q^{2}\right)_{n}} \cdot \frac{1-\zeta q^{2 n-1}}{1-\zeta / q} \cdot \frac{\left(\zeta ; q^{2}\right)_{n}^{2}}{\left(q ; q^{2}\right)_{n}^{2}} \cdot \frac{\left(t^{-1} ; q^{2}\right)_{n}}{\left(\zeta q t ; q^{2}\right)_{n}} t^{n} q^{n} \zeta^{-n} \\
=\frac{\left(\zeta q ; q^{2}\right)_{\infty}\left(\zeta^{-1} q ; q^{2}\right)_{\infty}}{\left(q ; q^{2}\right)_{\infty}\left(q ; q^{2}\right)_{\infty}}
\end{aligned}
$$

and in this form we see that it is a specialization of the limiting form of Jackson's theorem [6, p. 238, (II.20)].

To obtain (3.13) we note that

$$
(-\zeta)^{n}+(-\zeta)^{-n}= \begin{cases}2 & \text { if } n \equiv 0(\bmod 3), \\ -1 & \text { otherwise }\end{cases}
$$


Hence by Jacobi's triple product,

$$
\begin{aligned}
\frac{\left(\zeta q ; q^{2}\right)_{\infty}\left(\zeta^{-1} q ; q^{2}\right)_{\infty}}{\left(q ; q^{2}\right)_{\infty}^{2}} & =\frac{\left(q^{2} ; q^{2}\right)_{\infty}\left(\zeta q ; q^{2}\right)_{\infty}\left(\zeta^{-1} q ; q^{2}\right)_{\infty}}{\left(q^{2} ; q^{2}\right)_{\infty}\left(q ; q^{2}\right)_{\infty}^{2}} \\
& =\frac{\sum_{n=-\infty}^{\infty}(-1)^{n} q^{n^{2}} \zeta^{n}}{\sum_{n=-\infty}^{\infty}(-1)^{n} q^{n^{2}}} \\
& =\frac{1+\sum_{n=1}^{\infty} q^{n^{2}}\left((-\zeta)^{n}+(-\zeta)^{-n}\right)}{1+2 \sum_{n=1}^{\infty}(-1)^{n} q^{n^{2}}} \\
& =\frac{\sum_{n=-\infty}^{\infty} q^{(3 n)^{2}}-\sum_{n=-\infty}^{\infty} q^{(3 n+1)^{2}}}{1+2 \sum_{n=1}^{\infty}(-1)^{n} q^{n^{2}}}
\end{aligned}
$$

From (3.13) we immediately deduce a computationally fast recurrence for $\operatorname{PDO}(n)$.

COROllary 9 . For $n \geq 0$,

$$
\operatorname{PDO}(n)+2 \sum_{j \geq 1} \operatorname{PDO}\left(n-j^{2}\right)(-1)^{j}= \begin{cases}0 & \text { if } n \text { is not a square } \\ 1 & \text { if } n=0 \\ 2 & \text { if } n=(3 m)^{2} \\ -1 & \text { otherwise. }\end{cases}
$$

COROLlaRY 10. $\mathrm{PDO}(n)$ is odd precisely when either $n$ is 0 or a square not divisible by 3 .

Proof. By (3.13),

$$
\operatorname{pdo}(q) \equiv 1-\sum_{n=-\infty}^{\infty} q^{(2 n+1)^{2}}(\bmod 2) .
$$

4. Relations to other partition functions. In this section we shall consider three classical partition functions with an extensive literature.

$M(q)$ will denote the generating function for partitions without 1's and without any two parts differing by 1 . It is a theorem of MacMahon [12, p. 54] (see also [1]) that

$$
M(q)=\prod_{n \neq \pm 0,1(\bmod 6)}^{\infty}\left(1-q^{n}\right)^{-1}=\frac{\left(-q^{3} ; q^{3}\right)_{\infty}}{\left(q^{2} ; q^{2}\right)_{\infty}} .
$$

$S(q)$ will denote the generating function for partitions in which parts differ by at least 3 and multiples of 3 differ by at least 4 . Schur [16] has shown that

$$
S(q)=\prod_{n \neq \pm 2,3(\bmod 6)}^{\infty}\left(1-q^{n}\right)^{-1}=\frac{(-q ; q)_{\infty}}{\left(-q^{3} ; q^{3}\right)_{\infty}} .
$$


Our final generating function, $\Phi_{2}(q)$, is related to more complicated objects, generalized Frobenius partitions, and we refer the reader to [3] for an introduction to this topic. $\Phi_{2}(q)$ is the generating function for generalized Frobenius partitions wherein any number can appear on each row as a part at most twice. In $[3,(5.11)]$, it is shown that

$$
\begin{aligned}
\Phi_{2}(q) & =\prod_{n=1}^{\infty} \frac{1}{\left(1-q^{n}\right)\left(1-q^{12 n-10}\right)\left(1-q^{12 n-2}\right)\left(1-q^{12 n-3}\right)\left(1-q^{12 n-2}\right)} \\
& \left.=\frac{\sum_{m=-\infty}^{\infty} q^{(3 m)^{2}}-\sum_{m=-\infty}^{\infty} q^{(3 m+1)^{2}}}{(q ; q)_{\infty}^{2}} \quad \text { (by }[3,(10.4)]\right) .
\end{aligned}
$$

Given the above generating functions, we can easily combine these formulas with those of Sections 2 and 3 to deduce the following:

Theorem 11. For $|q|<1$,

$$
\begin{aligned}
\operatorname{pd}(q)(q ; q)_{\infty} & =M(q), \\
\operatorname{pd}(q) S(q) & =\frac{1}{(q ; q)_{\infty}^{2}}, \\
\operatorname{pdo}(q) & =\left(q^{2} ; q^{2}\right)_{\infty} \Phi_{2}(q) .
\end{aligned}
$$

Proof. Identity (4.3) follows from (4.1) and (1.3); identity (4.4) follows from (4.2) and (1.3). Finally identity (4.5) follows from (4.3) and (3.13).

It should be observed that these relationships are quite simple and suggest the possibility of our obtaining insight for partitions with designated summands from these classical partition functions.

5. Arithmetic properties. Because of their relationships with eta functions and modular forms, partition functions typically feature many interesting arithmetic properties. Our work on partitions with designated summands has produced infinite families of partitions whose generating functions are products of eta functions, and in this section we will give a sample of some divisibility and congruence properties for some of these partition functions. To work in the context of modular forms, we require the following facts:

Proposition 12 (Gordon, Hughes, Newman, Ligozat [8], [11], [14]). Let

$$
f(z)=\prod_{1 \leq \delta \mid N} \eta^{r_{\delta}}(\delta z)
$$

be a product of eta functions which satisfies the following criteria:

$$
\sum_{\delta \mid N} \delta r_{\delta} \equiv 0(\bmod 24)
$$


(ii)

$$
\sum_{\delta \mid N} \frac{N}{\delta} r_{\delta} \equiv 0(\bmod 24)
$$

(iii) For each $d \mid N$,

$$
\sum_{\delta \mid N}(d, \delta)^{2} \frac{r_{\delta}}{\delta} \geq 0
$$

Then $f(z) \in M_{k}\left(\Gamma_{0}(N)\right.$, $\left.\chi\right)$ if $k=\frac{1}{2} \sum r_{\delta} \in \mathbb{N}$ and $\chi$ is the quadratic character defined by

$$
\chi(l)=\left(\frac{(-1)^{k} \prod \delta^{r_{\delta}}}{l}\right) .
$$

Here $M_{k}\left(\Gamma_{0}(N), \chi\right)$ is the $\mathbb{C}$-vector space of holomorphic modular forms of weight $k$, level $N$, and Nebentypus character $\chi$. Conditions (i)-(ii) ensure that $f\left(\frac{a z+b}{c z+d}\right)=\chi(d)(c z+d)^{k} f(z)$ for all $z$ with $\operatorname{Im}(z)>0$ and for all integers $a, b, c, d$ such that $a d-b c=1$ and $N \mid c$. Condition (iii) guarantees that $f$ is "holomorphic at the cusps." For more on the basic theory of modular forms, see Koblitz [10]. We recall the following well-known facts about modular forms and their Fourier expansions.

Proposition 13 [10]. Suppose that $f(z) \in M_{k}\left(\Gamma_{0}(N), \chi\right)$ with Fourier expansion $f(z)=\sum_{n=0}^{\infty} a(n) q^{n}$. For any positive integer $t$,

$$
f(z) \mid T(t):=\sum_{n=0}^{\infty} \sum_{d \mid \operatorname{gcd}(t, n)} \chi(d) d^{k-1} a\left(\frac{t n}{d^{2}}\right) q^{n}
$$

is the Fourier expansion of a modular form in $M_{k}\left(\Gamma_{0}(N), \chi\right)$.

$$
f(z) \mid V(t):=\sum_{n=0}^{\infty} a(n) q^{t n}
$$

is the Fourier expansion of a modular form in $M_{k}\left(\Gamma_{0}(t N), \chi\right)$.

The operators $T(t)$ are the Hecke operators for integral weight modular forms. Note that as a special case of part (i) of this proposition we find that for any $t \mid N$,

$$
f(z) \mid U(t):=\sum_{n=0}^{\infty} a(t n) q^{n}
$$

is the Fourier expansion of a modular form in $M_{k}\left(\Gamma_{0}(N), \chi\right)$.

Proposition 14 [18]. Suppose $f(z)=\sum_{n=0}^{\infty} a(n) q^{n} \in M_{k}\left(\Gamma_{0}(N), \chi\right)$ satisfies:

(i) $a(n) \in \mathbb{Z}$ for all $n$,

(ii) $a(n) \equiv 0(\bmod M)$ for all $n \leq 1+(k N / 12) \prod_{p \mid N}(1+1 / p)$.

Then $a(n) \equiv 0(\bmod M)$ for all $n$. 
Lemma 15. Suppose that $f=\sum_{n=0}^{\infty} a(n) q^{n}, g=\sum_{n=0}^{\infty} d(m n) q^{m n}$ with $d(0)=1$, and

$$
f g=\sum_{n=0}^{\infty} b(n) q^{n}
$$

Then:

(i) If $a(m n+r) \equiv 0(\bmod M)$ for $0 \leq n \leq B$, then $b(m n+r) \equiv 0$ $(\bmod M)$ for $0 \leq n \leq B$.

(ii) If $b(m n+r) \equiv 0(\bmod M)$ for all $n$, then $a(m n+r) \equiv 0(\bmod M)$ for all $n$.

Proof. Both facts follow quickly after writing the coefficient of $q^{m n+r}$ from $f g$ in two ways:

$$
b(m n+r)=a(m n+r)+\sum_{k \geq 1} d(m k) a(m(n-k)+r) .
$$

Part (i) is obvious and part (ii) comes from a simple induction argument.

We begin with a theorem about the divisibility of $\mathrm{PD}^{p}(n)$ when $p$ is prime. The notation $\mathrm{PD}^{p}(n)$ is for the number of partitions of $n$ with designated summands wherein no part is divisible by $p$. Recall that we say almost all natural numbers have a property $P$ if

$$
\lim _{N \rightarrow \infty} \frac{\#\{n \leq N: P(n)\}}{N}=1 .
$$

THEOREM 16. If $p$ is any prime and $r$ any nonnegative integer, then almost all $n$ have the property that $\mathrm{PD}^{p}(n)$ is divisible by $p^{r}$.

Proof. It is a theorem of Serre [17] that for any positive integer $m$, almost all of the Fourier coefficients of an integer weight holomorphic modular form are divisible by $m$. Hence the theorem will follow if we can find, for each $p$ and sufficiently large $r$, modular forms $F_{r, p}(z)$ such that

$$
F_{r, p}(z) \equiv \sum_{n=0}^{\infty} \operatorname{PD}^{p}(n) q^{n}\left(\bmod p^{r}\right) .
$$

To this end we define, for all $r, p$ with $r \geq 1$ and $(p, 6)=1$,

$$
F_{r, p}(z):=\frac{\eta(6 z) \eta(p z) \eta(2 p z) \eta(3 p z) \eta^{p^{r}}(z)}{\eta(z) \eta(2 z) \eta(3 z) \eta(6 p z) \eta^{p^{r-1}}(p z)} .
$$

One can demonstrate that

$$
F_{r, p}(z) \in M_{p^{r-1}(p-1) / 2}\left(\Gamma_{0}(72 p), \chi\right)
$$


(where the character $\chi$ is defined in Proposition 12) by verifying the conditions of Proposition 12: It is easy to find that

$$
\sum_{\delta \mid 72 p} \delta r_{\delta}=0
$$

and

$$
\sum_{\delta \mid 72 p} \frac{72 p}{\delta} r_{\delta}=24\left(5-5 p+3 p^{r+1}-3 p^{r-1}\right) .
$$

For condition (iii), notice that for any $d \mid 72 p$,

$$
\sum \frac{(d, \delta)^{2} r_{\delta}}{\delta}
$$

depends only on $(d, 6 p)$. In the table below are shown the divisors of $6 p$ and the corresponding sums. They are all nonnegative, which completes the proof for $p \neq 2,3$.

\begin{tabular}{lc}
\hline$d \mid 6 p$ & $\sum(d, \delta)^{2} r_{\delta} / \delta$ \\
\hline$d=1$ & $5(1-p) /(3 p)+p^{r}-p^{r-2}$ \\
$d=2$ & $8(1-p) /(3 p)+p^{r}-p^{r-2}$ \\
$d=3$ & $3(1-p) / p+p^{r}-p^{r-2}$ \\
$d=6$ & $p^{r}-p^{r-2}$ \\
$d=p$ & $5(p-1) / 3$ \\
$d=2 p$ & $8(p-1) / 3$ \\
$d=3 p$ & $3(p-1)$ \\
$d=6 p$ & 0 \\
\hline
\end{tabular}

We shall omit the details for the cases $p=2,3$ which do not quite fit the argument above. Still it is routine to show that

$$
G_{r, 2}(z):=\frac{\eta^{2}(6 z) \eta(4 z) \eta^{2^{r}}(z)}{\eta(z) \eta(3 z) \eta(12 z) \eta^{2^{-1}}(2 z)} \equiv \sum_{n=0}^{\infty} \operatorname{PDO}(n) q^{n}\left(\bmod 2^{r}\right)
$$

is in $M_{2^{r-2}}\left(\Gamma_{0}(144), \chi_{\text {triv }}\right)$ for $r \geq 2$ and that

$$
H_{r, 3}(z):=\frac{\eta^{2}(6 z) \eta(9 z) \eta^{3^{r}}(z)}{\eta(z) \eta(2 z) \eta(18 z) \eta^{3^{r-1}}(3 z)} \equiv \sum_{n=0}^{\infty} \operatorname{PD}^{3}(n) q^{n}\left(\bmod 3^{r}\right)
$$

is in $M_{3^{r-1}}\left(\Gamma_{0}(108), \chi\right)$ for all $r$.

Although Theorem 16 already contains a strong statement about $\mathrm{PDO}(n)$ modulo powers of 2 , one can obtain more specific information using Hecke operators. For example, we find by an application of Sturm's criterion that

$$
\operatorname{pdo}(q) \mid T(p) \equiv 0(\bmod 4)
$$

for many small primes including $p=7,11,19,23,31$. This implies that

$$
\operatorname{PDO}(p n)-\operatorname{PDO}(n / p) \equiv 0(\bmod 4)
$$


for all such primes $p$ and all nonnegative integers $n$, which immediately gives infinitely many congruences in arithmetic progressions modulo 4 . The same techniques can be employed to produce specific information about any of the functions $\operatorname{pd}^{p}(q)$ modulo $p^{r}$, if desired.

Next we focus on some congruence properties of partitions with designated summands. We shall demonstrate a technique that can be used to computationally verify congruences for these partition functions and give some examples for the generating functions $\operatorname{pd}(q)$ and $\operatorname{pdo}(q)$. The first step is to combine Propositions 13 and 14 and Lemma 15 into a useful criterion. This technique was employed by Eichhorn and Ono [4] to prove congruence properties of modular functions.

TheOREM 17. Suppose that

$$
f(z):=\sum_{n=0}^{\infty} a(n) q^{n} \cdot \prod \eta^{r_{t \delta}}(t \delta z):=\sum_{n=0}^{\infty} r(n) q^{n} \in M_{k}\left(\Gamma_{0}(N), \chi\right)
$$

and that $a(t n-b) \equiv 0(\bmod M)$ for all

$$
n \leq B:=1+\frac{k N}{12} \prod_{p \mid N}\left(1+\frac{1}{p}\right)
$$

where $b=\left(\sum t \delta r_{t \delta}\right) / 24$. Then $a(t n-b) \equiv 0(\bmod M)$ for all $n$.

Proof. By the remark following Proposition 13,

$$
f(z) \mid U(t)=\sum_{n=0}^{\infty} r(t n) q^{n} \in M_{k}\left(\Gamma_{0}(N), \chi\right)
$$

so $r(t n) \equiv 0(\bmod M)$ for all $n$ if $r(t n) \equiv 0(\bmod M)$ for all $n \leq B$ by Proposition 14. Now, by the first part of Lemma 15, this will happen if $a(t n-b) \equiv 0(\bmod M)$ for all $n \leq B$, which in turn will imply that $a(t n-b) \equiv$ $0(\bmod M)$ for all $n$ by the second part of Lemma 15.

The above theorem successfully transfers Sturm's criterion from modular forms to other series under suitable conditions. We begin with one detailed argument.

COROLlary 18. For all nonnegative integers n,

$$
\mathrm{PD}(12 n+10) \equiv 0(\bmod 8) .
$$

Proof. Define the $\eta$-product $f(z)$ by

$$
\begin{aligned}
f(z) & :=\frac{\eta(6 z) \eta^{16}(12 z) \eta^{3}(24 z) \eta^{4}(36 z) \eta^{7}(72 z)}{\eta(z) \eta(2 z) \eta(3 z)} \\
& =\sum_{n=0}^{\infty} \operatorname{PD}(n) q^{n} \cdot \eta^{16}(12 z) \eta^{3}(24 z) \eta^{4}(36 z) \eta^{7}(72 z) \in M_{14}\left(\Gamma_{0}(72), \chi_{f}\right)
\end{aligned}
$$


according to Proposition 12. In the notation of Theorem 17,

$$
b=\frac{12 \cdot 16+3 \cdot 24+4 \cdot 36+7 \cdot 72}{24}=38
$$

and hence if $\mathrm{PD}(12 n-38) \equiv 0(\bmod 8)$ for all $n \leq 169$ then $\mathrm{PD}(12 n-38) \equiv 0$ $(\bmod 8)$ for all $n$. It has been verified computationally that $\operatorname{PD}(12 n+10) \equiv 0$ $(\bmod 8)$ for all $n \leq 165$, which implies the congruence (5.2) for all $n$.

We have found numerous congruences for partitions with designated summands which can be proven computationally using exactly the same argument as in Corollary 18. The work is in finding the right modular form $f(z)$ which implies the desired congruence by an application of Theorem 17 . Below we list many congruence properties and the weight $k$, level $N$, holomorphic modular forms $f(z)$ used to verify the congruences.

COROLlaRY 19. The following congruences are implied by the existence of the corresponding holomorphic modular forms $f(z)$ and a sufficient computation.

\begin{tabular}{ccc}
\hline Congruence & $f(z) \in M_{k}\left(\Gamma_{0}(N), \chi_{f}\right)$ & $(k, N)$ \\
\hline $\mathrm{PD}(24 n+18) \equiv 0(\bmod 24)$ & $\frac{\eta(6 z) \eta^{27}(24 z) \eta^{24}(48 z) \eta(72 z) \eta^{4}(144 z)}{\eta(z) \eta(2 z) \eta(3 z)}$ & $(27,144)$ \\
$\mathrm{PD}(12 n+6) \equiv 0(\bmod 4)$ & $\frac{\eta(6 z) \eta^{16}(12 z) \eta^{4}(24 z) \eta^{4}(36 z) \eta^{4}(72 z)}{\eta(z) \eta(2 z) \eta(3 z)}$ & $(13,72)$ \\
$\mathrm{PDO}(9 n+6) \equiv 0(\bmod 3)$ & $\frac{\eta^{2}(6 z) \eta(4 z) \eta^{8}(9 z) \eta^{8}(54 z) \eta^{2}(108 z)}{\eta(z) \eta(3 z) \eta(12 z)}$ & $(9,108)$ \\
$\mathrm{PDO}(12 n+10) \equiv 0(\bmod 3)$ & $\frac{\eta^{2}(6 z) \eta(4 z) \eta^{12}(12 z) \eta^{4}(24 z) \eta^{8}(48 z)}{\eta(z) \eta(3 z) \eta(12 z)}$ & $(12,48)$ \\
$\mathrm{PDO}(12 n+6) \equiv 0(\bmod 3)$ & $\frac{\eta^{2}(6 z) \eta(4 z) \eta^{12}(12 z) \eta^{12}(72 z)}{\eta(z) \eta(3 z) \eta(12 z)}$ & $(12,72)$ \\
$\mathrm{PDO}(24 n+16) \equiv 0(\bmod 3)$ & $\frac{\eta^{2}(6 z) \eta(4 z) \eta^{16}(24 z) \eta^{8}(48 z)}{\eta(z) \eta(3 z) \eta(12 z)}$ & $(12,48)$ \\
$\mathrm{PDO}(24 n) \equiv 0(\bmod 3)$ & $\frac{\eta^{2}(6 z) \eta(4 z) \eta^{24}(24 z)}{\eta(z) \eta(3 z) \eta(12 z)}$ & $(12,144)$ \\
\hline
\end{tabular}

There are undoubtedly many more simple congruences for the functions listed above and indeed for every member of our infinite families of generating functions which are $\eta$-products. The profusion of these arithmetic properties, especially for small moduli, suggests the likelihood of finding combinatorial explanations. Such a revelation could perhaps lead to the discovery and classification of families of congruences and a deeper understanding of partitions with designated summands.

6. Modular relations. In this section we consider the generating functions for $\mathrm{PD}(n)$ and $\mathrm{PDO}(n)$ in certain arithmetic progressions. We show that many of these generating functions are infinite products and also demonstrate that the phenomenon in Andrews' tenth problem $[3,5]$ on Frobenius 
partitions arises in the study of partitions with designated summands. Each of the Theorems 21-24 was observed by applying Euler's algorithm for infinite products [2, p. 98]. An application of Sturm's criterion guarantees that our observation was, in fact, a proof.

THEOREM 20.

$$
\begin{aligned}
\sum_{n=0}^{\infty} \operatorname{PD}(2 n) q^{n} & =\frac{\left(q^{4} ; q^{4}\right)_{\infty}^{2}\left(q^{6} ; q^{6}\right)_{\infty}^{5}}{(q ; q)_{\infty}^{3}\left(q^{2} ; q^{2}\right)_{\infty}\left(q^{3} ; q^{3}\right)_{\infty}^{3}\left(q^{12} ; q^{12}\right)_{\infty}^{2}}, \\
\sum_{n=0}^{\infty} \operatorname{PD}(2 n+1) q^{n} & =\frac{\left(q^{2} ; q^{2}\right)_{\infty}^{5}\left(q^{12} ; q^{12}\right)_{\infty}^{2}}{(q ; q)_{\infty}^{5}\left(q^{3} ; q^{3}\right)_{\infty}\left(q^{4} ; q^{4}\right)_{\infty}^{2}\left(q^{6} ; q^{6}\right)_{\infty}} .
\end{aligned}
$$

Proof. By Proposition 12, the equality

$$
\frac{\eta^{11}(2 z) \eta(6 z)}{\eta(z) \eta(3 z)}=\frac{\eta^{9}(2 z) \eta^{2}(8 z) \eta^{5}(12 z)}{\eta(4 z) \eta^{3}(6 z) \eta^{2}(24 z)}+\frac{\eta^{7}(2 z) \eta^{5}(4 z) \eta^{2}(24 z)}{\eta(6 z) \eta^{2}(8 z) \eta(12 z)}
$$

is an equality between modular forms in $M_{5}\left(\Gamma_{0}(72),\left(\frac{-1}{.}\right)\right)$ and hence is verified by comparing the first 61 terms on each side. Multiply both sides by $\eta^{-12}(2 z)$ to obtain

$$
\begin{aligned}
\sum_{n=0}^{\infty} \operatorname{PD}(2 n) q^{2 n}+\sum_{n=0}^{\infty} \operatorname{PD} & (2 n+1) q^{2 n+1} \\
= & \frac{\eta(6 z)}{\eta(z) \eta(2 z) \eta(3 z)} \\
= & \frac{\left(q^{8} ; q^{8}\right)_{\infty}^{2}\left(q^{12} ; q^{12}\right)_{\infty}^{5}}{\left(q^{2} ; q^{2}\right)_{\infty}^{3}\left(q^{4} ; q^{4}\right)_{\infty}\left(q^{6} ; q^{6}\right)_{\infty}^{3}\left(q^{24} ; q^{24}\right)_{\infty}^{2}} \\
& +q \cdot \frac{\left(q^{4} ; q^{4}\right)_{\infty}^{5}\left(q^{24} ; q^{24}\right)_{\infty}^{2}}{\left(q^{2} ; q^{2}\right)_{\infty}^{5}\left(q^{6} ; q^{6}\right)_{\infty}\left(q^{8} ; q^{8}\right)_{\infty}^{2}\left(q^{12} ; q^{12}\right)_{\infty}}
\end{aligned}
$$

and the theorem follows.

In an identical fashion we obtain Theorems 21 and 22 .

THEOREM 21.

$$
\begin{aligned}
\sum_{n=0}^{\infty} \operatorname{PDO}(2 n) q^{n} & =\frac{\left(q^{4} ; q^{4}\right)_{\infty}^{2}\left(q^{6} ; q^{6}\right)_{\infty}^{4}}{(q ; q)_{\infty}^{2}\left(q^{3} ; q^{3}\right)_{\infty}^{2}\left(q^{12} ; q^{12}\right)_{\infty}^{2}}, \\
\sum_{n=0}^{\infty} \operatorname{PDO}(2 n+1) q^{n} & =\frac{\left(q^{2} ; q^{2}\right)_{\infty}^{6}\left(q^{12} ; q^{12}\right)_{\infty}^{2}}{(q ; q)_{\infty}^{4}\left(q^{4} ; q^{4}\right)_{\infty}^{2}\left(q^{6} ; q^{6}\right)_{\infty}^{2}} .
\end{aligned}
$$

Proof. The equality

$$
\frac{\eta^{12}(2 z) \eta(4 z) \eta^{2}(6 z)}{\eta(z) \eta(3 z) \eta(12 z)}=\frac{\eta^{10}(2 z) \eta^{2}(8 z) \eta^{4}(12 z)}{\eta^{2}(6 z) \eta^{2}(24 z)}+\frac{\eta^{8}(2 z) \eta^{6}(4 z) \eta^{2}(24 z)}{\eta^{2}(8 z) \eta^{2}(12 z)}
$$


is an equality between modular forms in $M_{6}\left(\Gamma_{0}(144), \chi_{\text {triv }}\right)$ and hence is verified by checking the first 145 terms on each side. The claim follows immediately as in Theorem 20 after multiplying both sides by $\eta^{-12}(2 z)$.

THEOREM 22.

$$
\begin{aligned}
\sum_{n=0}^{\infty} \operatorname{PDO}(3 n) q^{n} & =\frac{\left(q^{2} ; q^{2}\right)_{\infty}^{2}\left(q^{6} ; q^{6}\right)_{\infty}^{4}}{(q ; q)_{\infty}^{4}\left(q^{12} ; q^{12}\right)_{\infty}^{2}} \\
\sum_{n=0}^{\infty} \operatorname{PDO}(3 n+1) q^{n} & =\frac{\left(q^{2} ; q^{2}\right)_{\infty}^{4}\left(q^{3} ; q^{3}\right)_{\infty}^{3}\left(q^{12} ; q^{12}\right)_{\infty}}{(q ; q)_{\infty}^{5}\left(q^{4} ; q^{4}\right)_{\infty}\left(q^{6} ; q^{6}\right)_{\infty}^{2}}, \\
\sum_{n=0}^{\infty} \operatorname{PDO}(3 n+2) q^{n} & =2 \cdot \frac{\left(q^{2} ; q^{2}\right)_{\infty}^{3}\left(q^{6} ; q^{6}\right)_{\infty}\left(q^{12} ; q^{12}\right)_{\infty}}{(q ; q)_{\infty}^{4}\left(q^{4} ; q^{4}\right)_{\infty}} .
\end{aligned}
$$

Proof. The equality

$$
\begin{aligned}
\frac{\eta^{7}(3 z) \eta(4 z) \eta^{2}(6 z)}{\eta(z) \eta(12 z)}= & \frac{\eta^{4}(3 z) \eta^{2}(6 z) \eta^{4}(18 z)}{\eta^{2}(36 z)}+\frac{\eta^{3}(3 z) \eta^{4}(6 z) \eta^{3}(9 z) \eta(36 z)}{\eta(12 z) \eta^{2}(18 z)} \\
& +2 \cdot \frac{\eta^{4}(3 z) \eta^{3}(6 z) \eta(18 z) \eta(36 z)}{\eta(12 z)}
\end{aligned}
$$

is easily seen by Proposition 12 to be an equality between modular forms in $M_{4}\left(\Gamma_{0}(144), \chi_{\text {triv }}\right)$ and hence is verified by comparing the first 97 terms on each side. Multiply both sides by $\eta^{-8}(3 z)$ and the theorem follows.

The generating functions for $\operatorname{PD}(3 n+0,1,2)$ do not have the same nice properties as in the previous theorems. However, in one case we find something striking.

Theorem 23. Define numbers a $(n)$ uniquely by

$$
\sum_{n=0}^{\infty} \operatorname{PD}(3 n) q^{n}=\prod_{n=1}^{\infty}\left(1-q^{n}\right)^{-a(n)} .
$$

Then for all positive $n$ we have

$$
a(6 n+1)=5, \quad a(6 n+3)=2, \quad a(6 n+5)=5 .
$$

We remark that the sequence $a(2 n)$ begins

$$
13,-14,80,-338,1741,-8902,16669, \ldots
$$

Proof of Theorem 23. Our object is to show that

$$
\sum_{n=0}^{\infty} \mathrm{PD}(3 n) q^{n}=\left(q ; q^{6}\right)_{\infty}^{-5}\left(q^{3} ; q^{6}\right)_{\infty}^{-2}\left(q^{5} ; q^{6}\right)_{\infty}^{-5} \cdot F\left(q^{2}\right)
$$

for some series $F$. By Proposition 12, we know that the $\eta$-product

$$
f(z):=\frac{\eta^{27}(6 z) \eta^{4}(3 z)}{\eta(z) \eta(2 z) \eta^{3}(9 z)}
$$


is a modular form in $M_{13}\left(\Gamma_{0}(18), \chi_{f}\right)$, and so is $g(z):=f(z) \mid U(3)$. Moreover,

$$
h(z):=g(z)-g(z)|U(2)| V(2)
$$

is a modular form in $M_{13}\left(\Gamma_{0}(36), \chi_{f}\right)$. An application of Sturm's criterion shows that $h(z)=0$. In other words, $g(z)=\sum_{n \geq 1} c(n) q^{2 n}$. To complete the proof, observe that

$$
\begin{aligned}
g(z) & =\frac{\eta^{27}(6 z) \eta^{4}(3 z)}{\eta(z) \eta(2 z) \eta^{3}(9 z)} \mid U(3) \\
& =\operatorname{pd}(q) \mid U(3) \cdot q^{2}\left(q^{2} ; q^{2}\right)_{\infty}^{26} \cdot \frac{(q ; q)_{\infty}^{5}}{\left(q^{3} ; q^{3}\right)_{\infty}^{3}}
\end{aligned}
$$

and therefore

$$
\begin{aligned}
\sum_{n=0}^{\infty} \operatorname{PD}(3 n) q^{n} & =\operatorname{pd}(q) \mid U(3)=\frac{\left(q^{3} ; q^{3}\right)_{\infty}^{3}}{(q ; q)_{\infty}^{5}} \cdot \frac{g(z)}{q^{2}\left(q^{2} ; q^{2}\right)_{\infty}^{26}} \\
& =\left(q ; q^{6}\right)_{\infty}^{-5}\left(q^{3} ; q^{6}\right)_{\infty}^{-2}\left(q^{5} ; q^{6}\right)_{\infty}^{-5} \cdot F\left(q^{2}\right) .
\end{aligned}
$$

7. Conclusion. It should be clear that partitions with designated summands are objects with a great deal of structure. It has not been our purpose to give an exhaustive account, but to offer a sample of their combinatorial, functional, and arithmetic properties. By using various tagging schemes, such as colored tags and restrictions on which parts can be designated, one can obtain many more families of infinite product generating functions. These undoubtedly have as many interesting properties as those discussed here, and in specific cases they have further relationships with generalized Frobenius partitions as well as with other types of partitions with designated summands. Since all of these have generating functions which are essentially eta functions, it should be worthwhile to consider the connections between partitions with designated summands and other objects which are counted by Fourier coefficients of modular forms.

\section{References}

[1] G. E. Andrews, A generalization of a partition theorem of MacMahon, J. Combin. Theory 3 (1967), 100-101.

[2] - The Theory of Partitions, Encyclopedia Math. Appl. 2, Addison-Wesley, Reading, 1976. [Reissued: Cambridge Univ. Press, Cambridge, 1985, paperback, 1998.]

[3] -, Generalized Frobenius partitions, Mem. Amer. Math. Soc. 301 (1984).

[4] D. Eichhorn and K. Ono, Congruences for partition functions, in: Analytic Number Theory, Vol. 1, Progr. Math. 138, Birkhäuser, 1996, 309-321.

[5] F. Garvan, Modular functions, maple and Andrews' 10th. problem, in: Topics in Number Theory, S. D. Ahlgren, G. E. Andrews and K. Ono (eds.), Kluwer, 1999, 163-179. 
[6] G. Gasper and M. Rahman, Basic Hypergeometric Series, Encylopedia Math. Appl. 35, Cambridge Univ. Press, Cambridge, 1990.

[7] J. W. L. Glaisher, Expressions for the first five powers of the series in which the coefficients are the sums of the divisors of the exponents, Messenger of Math., No. 171, July 1885.

[8] B. Gordon and K. Hughes, Multiplicative properties of $\eta$-products II, in: Contemp. Math. 143, Amer. Math. Soc., 1993, 415-430.

[9] G. H. Hardy and E. M. Wright, An Introduction to the Theory of Numbers, 4th ed., Oxford Univ. Press, London, 1960.

[10] N. Koblitz, Introduction to Elliptic Curves and Modular Forms, Springer, Berlin, 1984 .

[11] G. Ligozat, Courbes modulaires de genre 1, Bull. Soc. Math. France Mém. 43 (1975).

[12] P. A. MacMahon, Combinatory Analysis, Vol. 2, Cambridge Univ. Press, London, 1916. [Reprinted: Chelsea, New York, 1960.]

[13] - Divisors of numbers and their continuations in the theory of partitions, Proc. London Math. Soc. (2) 19 (1919), 75-113.

[14] M. Newman, Construction and application of a certain class of modular functions II, ibid. (3) 9 (1959), 353-387.

[15] S. Ramanujan, On certain arithmetical functions, Proc. Cambridge Philos. Soc. 22 (1916), 159-184. [Reprinted: Collected Papers of S. Ramanujan, Cambridge Univ. Press, Cambridge, 1927, 136-162; Reissued: Chelsea, New York, 1962.]

[16] I. Schur, Zur additiven Zahlentheorie, S.-B. Preuss. Akad. Wiss. Phys.-Math. Kl. 1926, 488-495. [Reprinted: I. Schur, Gesammelte Abhandlungen, Vol. 3, Springer, Berlin, 1973, 43-50.]

[17] J. P. Serre, Divisibilité de certaines fonctions arithmétiques, Enseign. Math. 22 (1976), 227-260.

[18] J. Sturm, On the congruence properties of modular forms, Lecture Notes in Math. 1240, Springer, Berlin, 1984, 275-280.

Department of Mathematics

Pennsylvania State University

University Park, PA 16802, U.S.A.

E-mail: andrews@math.psu.edu

Projet Théorie des Nombres

Institut de Mathématiques de Jussieu

Case 247

4 Place Jussieu

75252 Paris Cedex 05, France

E-mail: lovejoy@math.jussieu.fr
School of Mathematical Sciences

The University of Sussex

Brighton BN1 9QH, U.K.

E-mail: R.P.Lewis@sussex.ac.uk

Received on 6.4.2000

and in revised form on 7.1.2002 